\title{
DERIVING COMMON SEASONAL SIGNALS IN GPS POSITION TIME SERIES BY USING MULTICHANNEL SINGULAR SPECTRUM ANALYSIS
}

\author{
Marta GRUSZCZYNSKA ${ }^{1) *}$, Anna $\operatorname{KLOS}^{1)}$, Severine ROSAT ${ }^{2)}$ and Janusz BOGUSZ ${ }^{1)}$ \\ ${ }^{1)}$ Military University of Technology, Faculty of Civil Engineering and Geodesy, Warsaw, Poland \\ ${ }^{2)}$ Institut de Physique du Globe de Strasbourg, UMR 7516, Université de Strasbourg/EOST, CNRS, Strasbourg, France
}

*Corresponding author's e-mail: marta.gruszczynska@wat.edu.pl

\begin{tabular}{l} 
ARTICLE INFO \\
\hline Article history: \\
Received 3 January 2017 \\
Accepted 6 April 2016 \\
Available online 25 April 2017 \\
\hline
\end{tabular}

Keywords:

Multichannel Singular Spectrum Analysis Seasonal signals

GPS

ITRF2014

\begin{abstract}
We estimated the common seasonal signal (annual oscillation) included in the Global Positioning System (GPS) vertical position time series by using Multichannel Singular Spectrum Analysis (MSSA). We employed time series from 24 International GNSS Service (IGS) stations located in Europe which contributed to the newest ITRF2014 (International Terrestrial Reference Frame). The MSSA method has an advantage over the traditional modelling of seasonal signals by the Least-Squares Estimation (LSE) and Singular Spectrum Analysis (SSA) approaches because it can extract time-varying and common seasonal oscillations for stations located in the considered area. Having estimated the annual curve with LSE, we may make a misfit of $3 \mathrm{~mm}$ when a peakto-peak variations of seasonal signals are to be estimated due to the time-variability of seasonal signal. A variance of data modelled as annual signal with SSA and MSSA differs of $3 \%$ at average what proves that the MSSA-curves contain only time-varying and common seasonal signal and leave the station-specific part, local phenomena and power-law noise intact. In contrast to MSSA, these effects are modelled by SSA. The differences in spectral indices of power-law noise between MSSA and LSE estimated with Maximum Likelihood Estimation (MLE) are closer to zero than the ones between SSA and LSE, which means that MSSA curves do not contain site-specific noise as much as the SSA curves do.
\end{abstract}

\section{INTRODUCTION AND MOTIVATION}

Mathematical model of Global Navigation Satellite System (GNSS) position time series is composed of the initial value, linear trend, seasonal signals and stochastic part. Seasonal changes stem from real geophysical effects and artefacts (e.g. Dong et al., 2002; Wu et al., 2003; Ray et al., 2008; Collilieux et al., 2012). Dong et al. (2002) grouped the main contributors to seasonal signals in Global Positioning System (GPS) time series into three categories. The first one arises from gravitational excitation. The second category includes the phenomena associated with thermal expansion of bedrock and wind shear. Also, part of this category are atmospheric pressure, non-tidal oceanic changes and continental water storage. The last is related to various system errors that cause noticeable variations in estimated station's positions. Penna and Stewart (2003), Stewart et al. (2005) and Penna et al. (2007) proved that each un- or mismodelled sub-daily variability can propagate to several spurious longperiod features in GPS time series. Nowadays, it is widely acknowledged, that part of seasonal oscillations results from surface mass loading (e.g.
Blewitt et al., 2001; van Dam et al., 2001; Dong et al., 2002; van Dam et al., 2007; Tregoning et al., 2009; Davis et al., 2012; Jiang et al., 2013). Dong et al. (2002) showed that approximately $40 \%$ of the observed annual oscillations in the Up component may be explained by the joint contribution of pole tide effects and various loadings (ocean tidal and nontidal, atmospheric or ground water). Klos et al. (2017) proved that environmental loading models (atmospheric, hydrological and ocean non-tidal) are characterized by annual signal of amplitudes of up to $12 \mathrm{~mm}$ in vertical direction.

In general approach, seasonal oscillations are represented by the sum of sinusoids with annual and semi-annual cycles which are usually modeled by using Least Squares Estimation (LSE) (Blewitt et al., 2001; Collilieux et al., 2007; van Dam et al., 2007; Bogusz and Figurski, 2014; Bogusz and Klos, 2016), which means that obtained seasonal signals are characterized by the constant amplitudes and phases in time. In fact, real seasonal signals observed by GPS are modulated over time. Bennett (2008) proved that seasonal oscillations are characterized by timevariable amplitude and that neglecting the decadal 
variation may cause the bias of velocity estimates. Freymueller (2009), Tesmer et al. (2009) and Bogusz et al. (2016) proposed to use non-parametric methods as stacking and clustering to determine time-varying annual oscillations from GPS time series. Davis et al. (2012) applied a Kalman filter approach to extract seasonal variability. They explored implications for modelling and noise analysis of stochastic seasonal processes by studying the form of Power Spectral Density (PSD). Recently, Xu (2016) used Cross Wavelet Transform (XWT) to examine the relationship between GPS height residuals and mass loadings. The XWT-based semblance analysis confirmed that the annual oscillations result from a combination of the geophysical signals and systematic errors.

The purpose of this research is to investigate the capability of Multichannel Singular Spectrum Analysis (MSSA) to determine common seasonal signal for a set of time series. The method we use is a natural extension of the classical Karhunen-Loève method (Karhunen, 1946; Loève, 1945) and it is based on the orthogonal transformation which is a popular choice for geoscience analysis to reduce data dimensionality. MSSA is also a special case of Singular Spectrum Analysis (SSA) accessible for multivariate time series. Up till now, methods based on the orthogonal transformation were used to deliver the time-varying curves. Zerbini et al. (2013) applied Empirical Orthogonal Functions (EOFs) and Singular Value Decomposition (SVD) to analyze data collected at the stations located in Europe. They investigated the inter-annual variability of GPS coordinates, atmospheric pressure, terrestrial water storage and gravity time series obtained with GRACE gravity mission. The SSA approach was applied to investigate the non-linear station motions from DORIS-derived time series (Khelifa et al., 2012) or to determine modulated seasonal variations within weekly GPS time series (Chen et al., 2013). In the latter, the authors proved that SSA has the ability to extract time-variable seasonal signals (annual and semiannual) and non-linear trend from GPS time series. More recently, Xu and Yue (2015) used Monte Carlo SSA (MCSSA) to extract the time-variable seasonal signal from daily GPS position time series and conducted statistical analysis on the colored noise. MSSA, which we employ in this research, has been only recently used in geodesy field. Rangelova et al. (2010) used MSSA to study the water mass changes for GRACE monthly data, and to model the glacial isostatic adjustment (GIA) signal. Thereafter, Rangelova et al. (2012) analyzed a 6-year, weekly GRACE time series and hydrological models, using MSSA to extract non-periodic mass variations. Zhu et al. (2016) applied MSSA to find the significant inter-annual oscillations in glacier mass by using GRACE data from Central Asia. They determined the inter-annual oscillations with period of 6.1 years and 2.3 years. Finally, Walwer et al. (2016) used the
MSSA approach to determine common seasonal signals and microdeformations at the Akutan active volcano from GPS data. They proved that MSSA is an appropriate tool to extract common oscillations from GPS position time series and to separate seasonal signals from noise. Against this background, the main question that motivates our research is whether MSSA is the optimal method to investigate the common seasonal oscillation in GPS time series and also, to deliver only those frequencies which are significant for a set of analysed stations, separating them from site-specific and local effects.

This research paper is organized as follows. Initially, in Section 2, we describe the detailed methodology of the MSSA. Section 3 is a short description of data we used in our research. In Section 4, we apply MSSA for GPS vertical position time series and present the results. We discuss the lag window size which is a key problem before implementing the MSSA approach. We investigate the possibility of extracting time-varying signals and common variations from a set of time series and underline the main properties of MSSA. Then, MSSA-derived signals are compared to the signals obtained by using LSE and SSA.

\section{MULTICHANNEL SINGULAR SPECTRUM ANALYSIS}

Multichannel (or Multivariate) Singular Spectrum Analysis (MSSA) is a non-parametric tool for simultaneous analysis of a set of time series characterized by common structures. This approach is based on the classical Karhunen-Loève expansion and it is an extension of the SSA method (Ghil et al., 2002; Broomhead and King, 1986a, 1986b; Allen and Robertson, 1996; Raynaud et al., 2005). Broomhead and King (1986b) were the first who proposed to make use of MSSA in the context of nonlinear dynamics. Over the last two decades, this approach has become a widely used tool in different fields e.g. climatology, oceanography, economics, biomedical sciences (Ghil et al., 2002; Allen and Robertson, 1996; Hassini and Zhigljavsky, 2009; Golyandina and Zhigljavsky, 2013; Zhang et al., 2014). As a univariate method, MSSA allows to decompose the time series into its spectral components.

The main advantage of this method over other methods (e.g. SSA and LSE) is the ability to extract common seasonal signals, common trends and common noises from multivariate time series. The method is based on the determination of the eigenvalues and the eigenvectors of the covariance matrix, and on the reconstruction of the time series.

As a natural extension of SSA, MSSA includes two complementary stages: decomposition and reconstruction. The starting point of MSSA is the embedding procedure. We have $L$ time series with $N$ data points. The window of length $M$ can be moved by each single observation for individual time series: 


$$
\left\{X_{l}(t): t=1, \ldots, N\right\}, 1 \leq l \leq L
$$

In this process, we receive a trajectory matrix for individual time series (Broomhead and King, 1986a, 1986b; Allen and Robertson, 1996; Ghil et al., 2002):

$$
\tilde{\mathbf{X}}_{l}=\left(\begin{array}{cccc}
X_{l}(1) & X_{l}(2) & \cdots & X_{l}(M) \\
X_{l}(2) & X_{l}(3) & \cdots & X_{l}(M+1) \\
\vdots & \vdots & \cdots & \vdots \\
X_{l}\left(N^{\prime}-1\right) & \vdots & \cdots & X_{l}(N-1) \\
X_{l}\left(N^{\prime}\right) & X_{l}\left(N^{\prime}+1\right) & \cdots & X_{l}(N)
\end{array}\right)
$$

The dimension of the trajectory matrix of each time series is $N^{\prime} \times M$, where $N^{\prime}=N-M+1$. The multichannel trajectory matrix $\tilde{\mathbf{D}}$ can be defined as:

$$
\tilde{\mathbf{D}}=\left(\tilde{\mathbf{X}}_{1}, \tilde{\mathbf{X}}_{2}, \ldots, \tilde{\mathbf{X}}_{L}\right)
$$

This full augmented trajectory matrix has $L \cdot M$ columns of length $N-M+1$ (Groth and Ghil, 2011). Using obtained trajectory matrix, the grand lagcovariance matrix $\tilde{\mathbf{C}}_{D}$ can be computed:

$$
\tilde{\mathbf{C}}_{D}=\frac{1}{N^{\prime}} \tilde{\mathbf{D}}^{t} \tilde{\mathbf{D}}=\left(\begin{array}{ccccc}
\mathbf{C}_{1,1} & \mathbf{C}_{1,2} & \cdots & \cdot & \mathbf{C}_{1, L} \\
\cdot & \mathbf{C}_{2,2} & \cdots & \cdot & \cdot \\
\cdot & & \cdots & \cdot & \cdot \\
\cdot & & \cdots & \mathbf{C}_{l, l^{\prime}} & \cdot \\
\cdot & & \cdots & \cdot & \cdot \\
\mathbf{C}_{L, 1} & \mathbf{C}_{L, 2} & \cdots & \cdot & \mathbf{C}_{L, L}
\end{array}\right)
$$

The dimension of the grand lag-covariance matrix is $(L \cdot M) \times(L \cdot M)$. Each of the element of the lag-covariance matrix is given by (Ghil et al., 2002):

$$
\mathbf{C}_{l, l^{\prime}}=\frac{1}{N^{\prime}} \tilde{\mathbf{X}}_{l}^{t} \tilde{\mathbf{X}}_{l^{\prime}}
$$

The method we applied to compute the grand lag-covariance matrix was proposed by Broomhead and King (1986a) and is known as the BK algorithm.

In the second step, we conducted the diagonalization of the grand lag-covariance matrix applying the Singular Value Decomposition (SVD) obtaining eigenvectors $\mathbf{E}^{k}$ (also called Empirical Orthogonal Functions - EOFs) and eigenvalues $\lambda_{k}$ by formula:

$$
\boldsymbol{\Lambda}=\mathbf{E}^{T} \mathbf{C} \mathbf{E}
$$

where:

$\Lambda$ - matrix which contains eigenvalues $\lambda_{k}$,

E - matrix which contains particular eigenvectors $\mathbf{E}^{k}$.

It is important that eigenpairs $\left(\lambda_{k}, \mathbf{E}^{k}\right)$ have to be sorted in descending order of eigenvalues. Using the obtained eigenvectors, we calculated the $k$-th Principal Components (PCs) which are a single-channel time series (Ghil et al., 2002):
$A^{k}(t)=\sum_{j=1}^{M} \sum_{l=1}^{L} X_{l}(t+j-1) E_{l}^{k}(j)$

Principal Components represent the projection of the original time series onto the eigenvectors.

The MSSA approach is an extension of classical Principal Component Analysis (PCA), but both of them differ in their character. In the PCA method $(M=1)$, a covariance matrix is simply a spatial covariance function of data. However, in MSSA, a covariance matrix is augmented by time-lagged covariance functions (Kim and $\mathrm{Wu}, 1999$ ). The main advantage of MSSA eigenvectors over PCA eigenvectors is the ability to identify oscillatory behaviour in time because of oscillatory pairs (two eigenvectors of a pair correspond to the same period) (Groth and Ghil, 2011).

According to Plaut and Vautard (1994), harmonic oscillations in time series can be identified by the following three properties of MSSA: (a) two consecutive eigenvalues explain almost the equal amount of variance, (b) two EOFs of a pair of modes correspond to almost the exact frequency and are in quadrature (sine and cosine), and (c) associated Principal Components are in quadrature.

The last step of the MSSA algorithm is a reconstruction procedure in order to compute Reconstructed Components (RCs) using previously calculated Principal Components and Empirical Orthogonal Functions. The $k$-th Reconstructed Components (RCs) at time $t$ for channel $l$ is defined by the following formulas (Plaut and Vautard, 1994):

$$
R_{l}^{k}(t)=\left\{\begin{array}{ccc}
\frac{1}{M} \sum_{j=1}^{M} A^{k}(t-j+1) E_{l}^{k}(j) & \text { for } & M \leq t \leq N-M+1 \\
\frac{1}{i} \sum_{j=1}^{M} A^{k}(t-j+1) E_{l}^{k}(j) & \text { for } & 1 \leq t<M-1 \\
\frac{1}{N-i+1} \sum_{j=1-N+M}^{M} A^{k}(t-j+1) E_{l}^{k}(j) & \text { for } & N-M+2 \leq t \leq N
\end{array}\right.
$$

In the MSSA process, if we sum up all the individual RCs, information is not lost. Nevertheless, the MSSA method is a special type of filtration if we choose particular RCs. In our study, these selected RCs were associated with annual signals.

\section{DATA}

We used daily GPS height time series from 24 stations located in Europe (Fig. 1). They were derived from Network Solution produced by the International GNSS Service (IGS) and contributed (Rebischung et al., 2016) to the newest realization of the International Terrestrial Reference System (namely ITRF2014, Altamimi et al., 2016). Employed position time series were not shorter than 10 years with the maximum length of 23 years. Initially, we conducted the pre-analysis to remove offsets, outliers and gaps. Offsets were removed using epochs defined by IGS. In order to detect and remove outliers we 


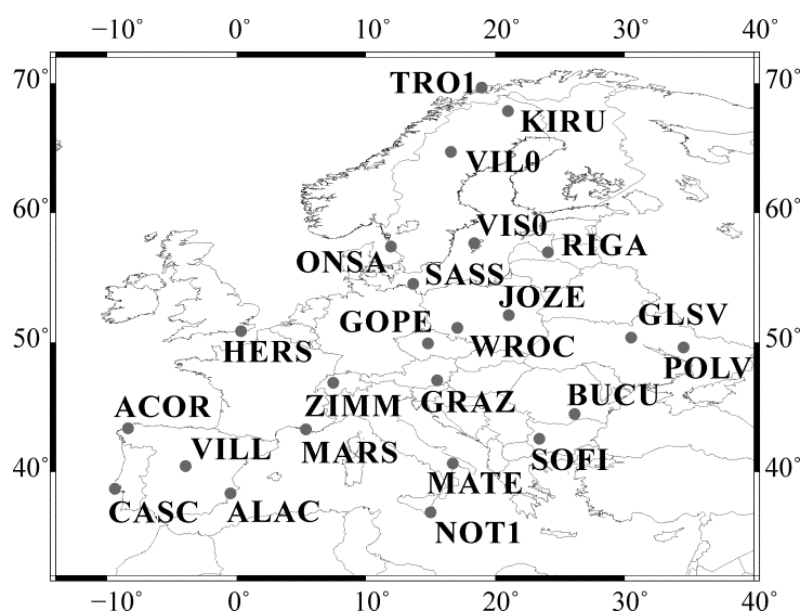

Fig. 1 Layout of the GPS permanent stations which position time series were employed to the analysis.

used the Interquartile Range rule (IQR). One of the requirements of either SSA or MSSA is that time series are continuous. Following that, the gaps in GPS time series were interpolated assuming a combination of white noise and mathematical model of series estimated with LSE. The scatter of time series (standard deviation) was iteratively determined in order to ensure that the variance of data was the same to that before interpolation. Adding the mathematical model caused that we did not introduce any artificial modulation in amplitude. MSSA also requires a common range of observations, thus a data time span ranging from 2003.08 to 2013.98 was chosen. In this research, we only focus on vertical changes of GPS position series as the time-variability of those data is the greatest. Naturally enough, presented methodology can be also successfully applied to horizontal changes.

\section{RESULTS}

\section{1. $M S S A$}

The MSSA approach has to be preceded by a choice of lag-window size $M$ that defines the ability to distinguish between two spectral peaks (Ragelova et al., 2012). If $M$ is too small, the coarse resolution may cause several neighbouring peaks in the spectrum of the signal (Vautard et al., 1992). However, too large $M$ values will split the peak into several components with neighbouring frequencies. Golyandiana and Zhigljavsky (2013) proposed to select a lag-window size $M$ that is an integer multiple of the period of interest, if it is recognized. In the case of SSA, Chen et al. (2013) proved that two or three years lag-window is suitable for most of GPS time series. In our previous research (Gruszczynska et al., 2016), we applied Akaike Information Criterion (AIC, Akaike, 1974) to choose the optimal lag-window size when the SSA technique was implemented. A 3-year length lag-window was optimal to determine timevarying annual oscillations. In the case of MSSA,

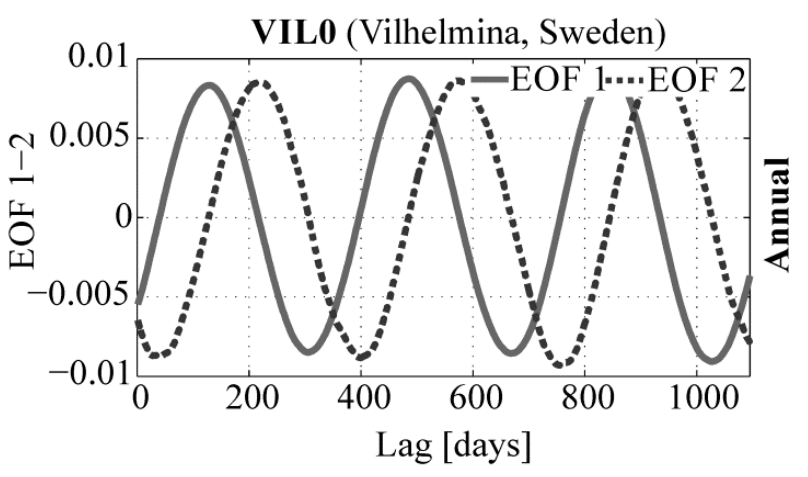

Fig. 2 The $1^{\text {st }}$ and $2^{\text {nd }}$ EOFs of the Up topocentric changes for the VIL0 station.

Rangelova et al. (2012) also chose a 3-year lagwindow (156 weeks) to extract annual and semiannual signals from the GRACE gravity field solutions. Walwer et al. (2015) chose a 400-day lagwindows to extract the annual signal from GPS time series.

In this research, we chose a 3-year lag-window size $M$ to determine the common seasonal oscillation (annual) for a set of employed stations. Figure 2 shows the $1^{\text {st }}$ and $2^{\text {nd }}$ EOFs which correspond to annual period for VIL0 (Vilhelmina, Sweden) station. These curves of Empirical Orthogonal Functions are in quadrature. Whereas, the $3^{\text {rd }}$ and $4^{\text {th }}$ EOFs have the frequency of 0.12 and 0.18 cpy (cycle per year), respectively. This means they contain the long-term changes or the non-linear long-term variability. They are located prior to semi-annual signal $\left(5^{\text {th }}\right.$ and $6^{\text {th }}$ EOFs) with a frequency of 2 cpy what means that their contribution to the common signal is higher than the one arising from semi-annual curve. This in turn means that the semi-annual signal may not be as evident for a set of employed stations as the annual curve is. In this study, we focus on annual signal $\left(1^{\text {st }}\right.$ and $2^{\text {nd }}$ EOFs), that is why we left out the $3^{\text {rd }}, 4^{\text {th }}$ and higher EOFs with similar variance. Their modelling falls outside the scopes of this paper.

Using the obtained eigenvectors, we calculated the $k$-th Principal Components (PCs) which are a single-channel time series. We applied the Welch's periodogram (Welch, 1967) to determine the frequency of particular eigenvalues. Figure 3 shows the $1^{\text {st }}$ and $2^{\text {nd }}$ PCs which are related to annual signal for all stations. Each of the Principal Components contains common characteristics of all employed time series. This property allows computing common seasonal signals for all stations. In fact, the curves of our interest have the frequency around 1 cpy (365.25 days). Due to insufficient length of data, the annual signal we modelled is a comb of all changes around one year. For example, to reliably resolve one tropical and one draconitic years in spectral domain, we would need 25.6 years of data. Consequently, in this research, whenever we mention annual signal, we mean all changes around one year. 


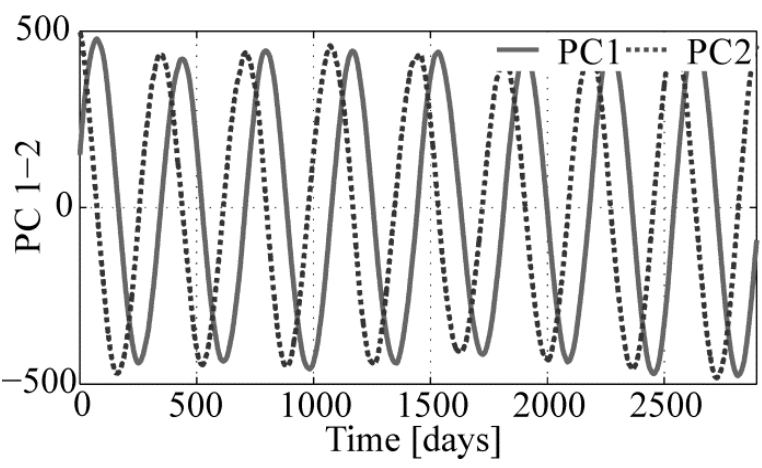

Fig. 3 The $1^{\text {st }}$ and $2^{\text {nd }}$ PCs of the Up topocentric changes for a set of employed stations. A slight modulation in annual changes can be noticed.

For each of the estimated Principal Component (representing the pattern of the common signal) we assigned the corresponding eigenvalue which represents the variance explained by this mode. Analysis of the particular eigenvalue increases the possibility to investigate and interpret the seasonal signals.

The Reconstructed Component (RC) can be created using Principal Components and Empirical Orthogonal Functions estimated previously. If we sum up all of the individual RCs, we will obtain the original time series. Each station's annual signal occurs in the $1^{\text {st }}$ and $2^{\text {nd }}$ Reconstructed Component due to the highest contribution into analysed signal. Figure 4 shows the first and the second Reconstructed Components (annual signal) for the Up component of VIL0 station. For all stations, common seasonal oscillations were estimated by summing up the $1^{\text {st }}$ and $2^{\text {nd }}$ Reconstructed Components.

Figure 5 represents the original time series and the reconstructed time series (annual oscillation) for VIL0 and BUCU (Bucuresti, Romania) stations. For

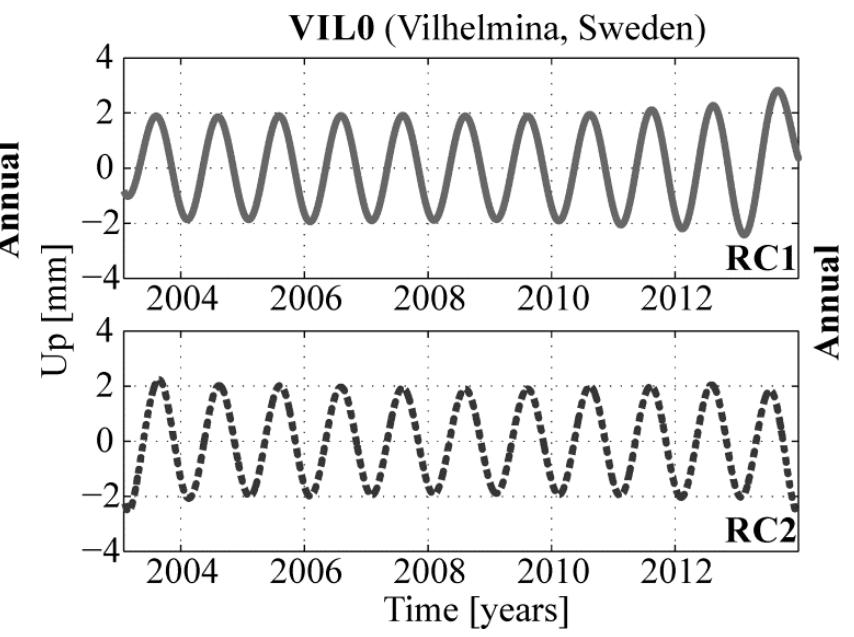

Fig. 4 The $1^{\text {st }}$ and $2^{\text {nd }}$ RCs of the Up topocentric changes for VIL0 station.

VIL0 station the estimated annual signal fits the original data very good for entire data. A slight modulation in amplitude of VIL0 series which was not covered by MSSA curve can be noticed between 2006 and 2010. This modulation may arise from local phenomena which is not reflected in a common seasonal signal modelled with MSSA. The filtered signal fits well the original time series of BUCU between 2009 and 2014. However, few discrepancies between model and original data can be noticed. Period of 2003-2007 is slightly overestimated by MSSA-curve, while period of 2007-2009 is a little bit underestimated. This might mean that BUCU is affected by local phenomena in the period of 20032009 which are in fact not reflected in other time series employed in this analysis. On the other hand, this lack of fit may likewise arise from a long-term non-linearity explained by $3^{\text {rd }}$ and $4^{\text {th }} \mathrm{RCs}$. Due to above, the MSSA-curve which contains common seasonal signals may not exactly match all changes within individual data, but is mainly created basing on common changes of employed data.
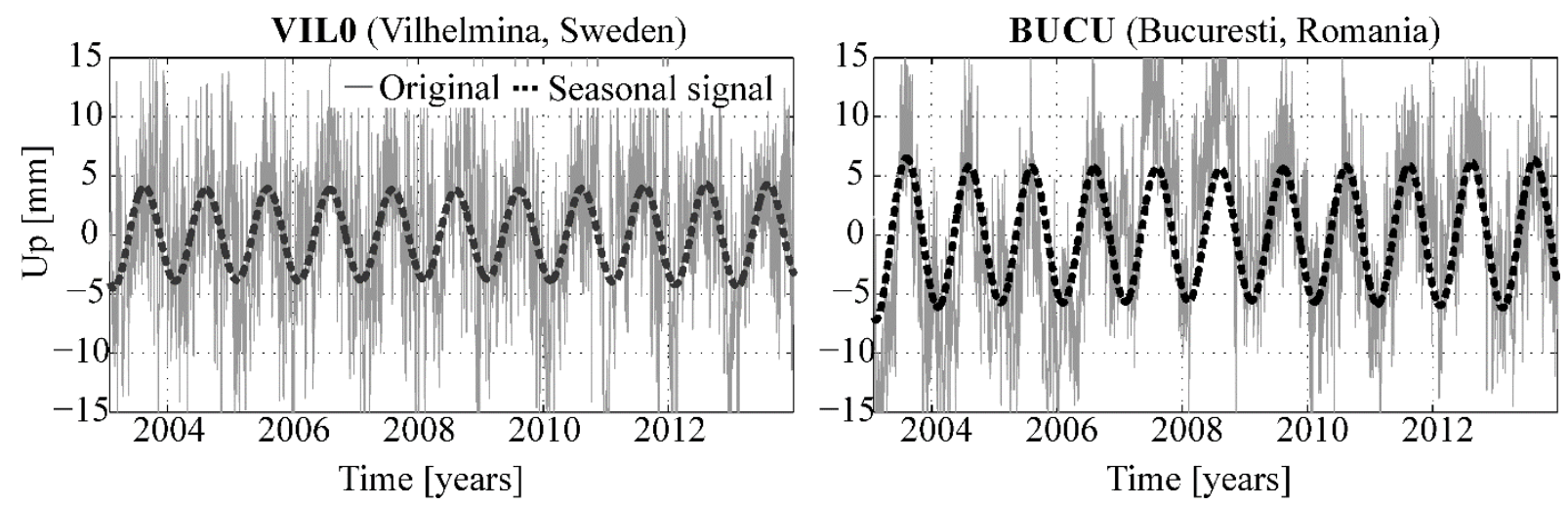

Fig. 5 Original time series (grey line) and MSSA-reconstructed seasonal signal (black line) for VIL0 (left) and BUCU (right) stations. 


\subsection{MSSA VS LSE}

To demonstrate the main properties of the MSSA method, we compared the seasonal signals for all analysed stations derived using MSSA and LSE. For the employed stations, the implementation of the MSSA approach allows to estimate common seasonal oscillation which is varying over time, whereas the LSE approach estimates seasonal curves individually for each station with no changes in their amplitude over time.

Figure 6a presents the stacked original detrended GPS height time series for all of employed stations, while Figure $6 \mathrm{~b}$ shows annual signal derived using LSE for all stations. The last one (Fig. 6c) presents the annual time-modulated signals determined using MSSA.

Due to the parametric character of the LSE approach, we can extract seasonal signals which are characterised by constant amplitudes and phases (Fig. 6b) which is the obvious disadvantage of this method. In reality, seasonal oscillations in geodetic time series are not constant over time (e.g. Chen et al., 2013). The results (Fig. 6c) reveal that MSSA can extract common time-varying seasonal pattern from time series. We observed that the time-changeable oscillations derived with MSSA are characterised by higher amplitude at the beginning and at the end of common data span. The positive peak value is equal to $7.7 \mathrm{~mm}$ in 2003.6 while, in 2003.1, the negative peak value is equal to $-8.3 \mathrm{~mm}$. If we estimated timeconstant oscillations, we may make a misfit of $3 \mathrm{~mm}$ when a peak-to-peak variations of seasonal signals are to be estimated.

\subsection{MSSA VS SSA}

As it was previously stated, MSSA is an extension of the SSA to the case of multivariate time series. The majority of the MSSA algorithm steps are similar to the steps of univariate approach. It is notable that this method differs in terms of the structures of the trajectory matrix and its approximation (Zhang et al., 2014). In the case of SSA, the trajectory matrix is a straightforward Hankel matrix, as for MSSA - a block Hankel matrix (Hassani and Mahmoudvand, 2013).

Figure 7 shows the variance of data explained by certain modes of SSA and MSSA for VIL0 (SSA) and all employed stations (MSSA). In case of VIL0, the modes of SSA present annual signal $\left(1^{\text {st }}\right.$ and $2^{\text {nd }}$ modes, period of 365.25 days), non-linear longterm trend $\left(3^{\text {rd }}\right.$ and $4^{\text {th }}$ modes, period of 3043.7 and 2029.2 days, respectively) and semi-annual signal $\left(5^{\text {th }}\right.$ and $6^{\text {th }}$ modes, period of 182.62 days). The periods of modes were estimated with Welch periodogram. The highest variance was found for annual signal, which contributes into the total variance of data in almost $23 \%$. Then, the non-linearity exceeds the semi-annual signal and contributes in almost $2 \%$ into the total variance of data. The non- linearity is followed by semi-annual signal and then, consecutively by noise. The difference in explained variance between by the $3^{\text {rd }}, 4^{\text {th }}, 5^{\text {th }}, 6^{\text {th }}$ and the consequtive modes is very small, therefore, we only determine the annual signal in this study. The SSAmodelled curves may contain real seasonal changes that are common for entire set of data we employed, but may also be affected by station-dependent or local oscillations which were not distinguished between certain modes. As can be easily noticed from Figure 7, we are not able to sufficiently resolve between semiannual signal and noise, which is following $5^{\text {th }}$ and $6^{\text {th }}$ modes. Or, the variance of noise is as high as the variance of semi-annual signal and therefore, some power may be filtered through consecutive modes. Summing up, the SSA-modelled curve will match all time-varying changes in individual series, but also may contain some part of noise. The MSSA-modelled curves due to the character of MSSA will contain only time-varying common signals that arise from real large-scale geophysical changes or artefacts related to the mismodelling of Earth Orientation Parameters (EOP) or satellite antenna phase centre (APC) and will leave the station-specific or local part intact. The investigation of the origin is out of the scope of this paper.

It can be noticed from Figure 7 that a percentage of variance modelled as annual signal with SSA and MSSA differs of $3 \%$. It means, that $3 \%$ of variance modelled as annual signal using SSA for VIL0 is a station-specific or a local annual variation due to local hydrology or any other local phenomena. In its turn, this percentage is not included and not explained by the joint annual signal estimated for a set of employed stations. Finally, the MSSA-modelled curves will not be affected by station-specific or local changes or station-specific character of power-law noise and therefore will only contain the joint seasonal changes.

In order to depict the main advantages of the MSSA method over SSA, we compared the computed signals for GPS height time series using the first ten Reconstructed Components. Original detrended GPS position time series are shown in Figure 8 in grey for VIL0 and VIS0 (Visby, Sweden) stations. The MSSAand SSA-modelled curves are plotted in black. Clearly noticeable is the fact that SSA-modelled curve contains both station individual signal and noise. Especially, VIS0 station is affected by clear noise when the Reconstructed Components from $1^{\text {st }}$ to $10^{\text {th }}$ are summed up. It is important to emphasize that the first Reconstructed Components reflect the highest variance regarding to the complete variability of each time series. MSSA-curves are much smoother than SSA since they focus only on common signal that can be observed for a set of series. In contrast to signals determined by using SSA, the first ten Reconstructed Components reflect the highest variance for selected set of time series. 

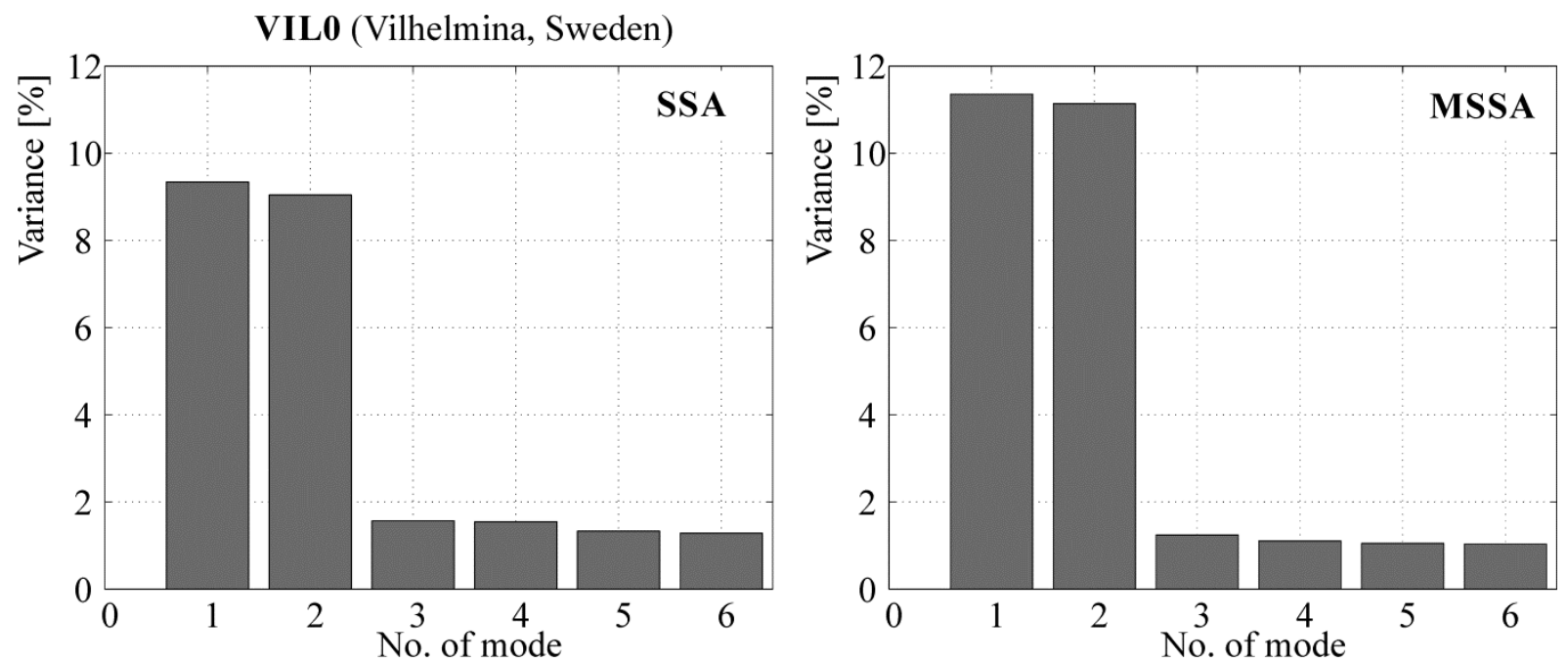

Fig. 7 Variance of data explained by certain modes of SSA for VIL0 station and MSSA for a set of employed stations.
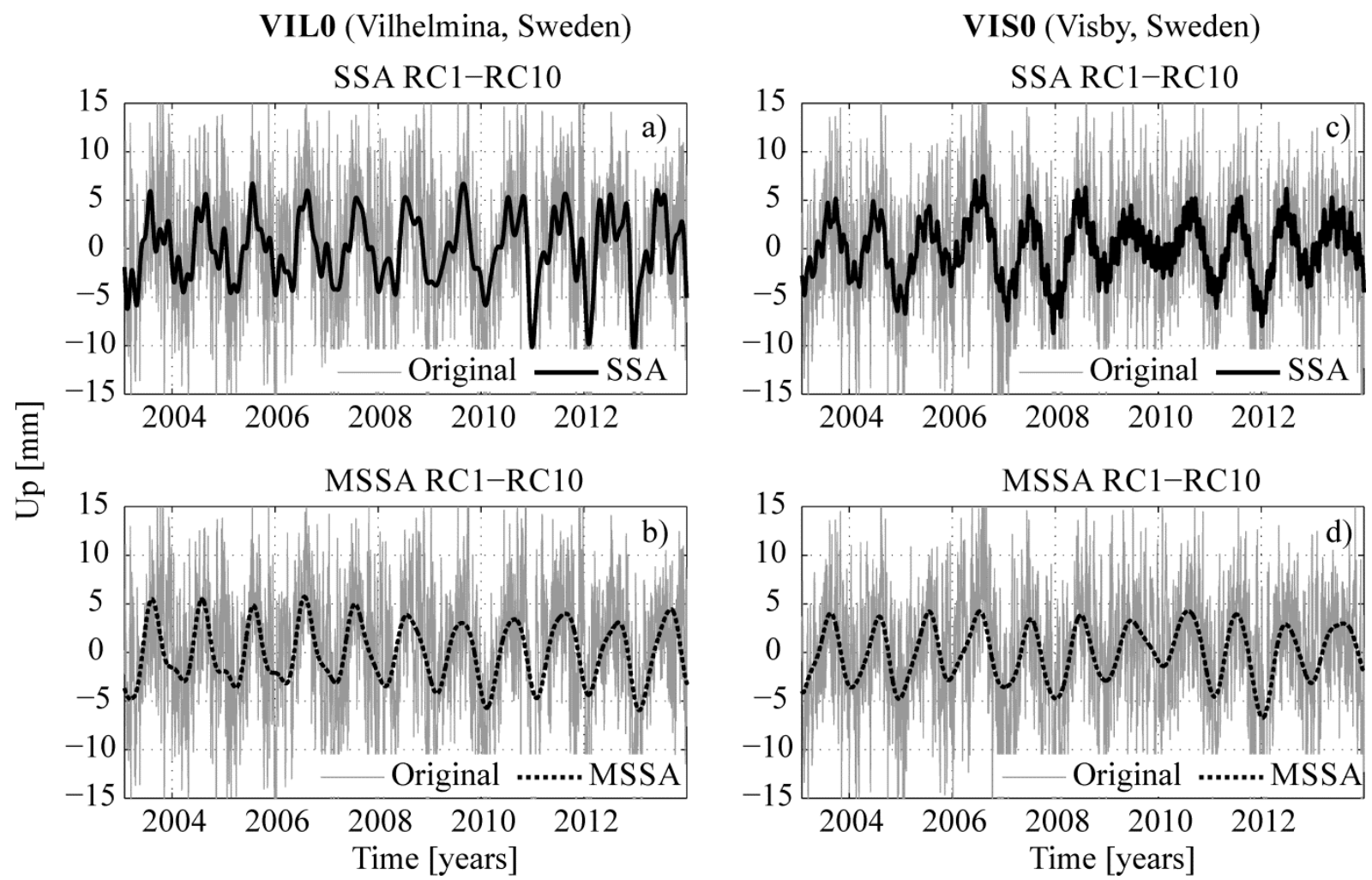

Fig. 8 Original detrended GPS time series are represented in grey for VIL0 and VIS0 station in the Up component. Then, reconstructions of the signals using the first 10 components for SSA (a, c) and MSSA $(b, d)$ are presented.

Figure 9 presents the PSD estimated with Welch algorithm for BUCU and VIL0 station. Power of original data is plotted in black, powers estimated after LSE- (blue), SSA- (brown) and MSSA-modelled (red) curves were removed are also plotted on the top of original data. Original data shows evident oscillations of 1 and 2 cpy. Power of residuals after LSE was implemented shows a reduction of peaks of one year. However, both curves are estimated as being constant over time. Both SSA and MSSA provide time changeable curves. SSA removes more power from the seasonal frequency band than MSSA. This arises from the fact that the SSA approach models each of time series separately. Therefore, all local effects which leaked through consecutive modes are included in the reconstruction. 

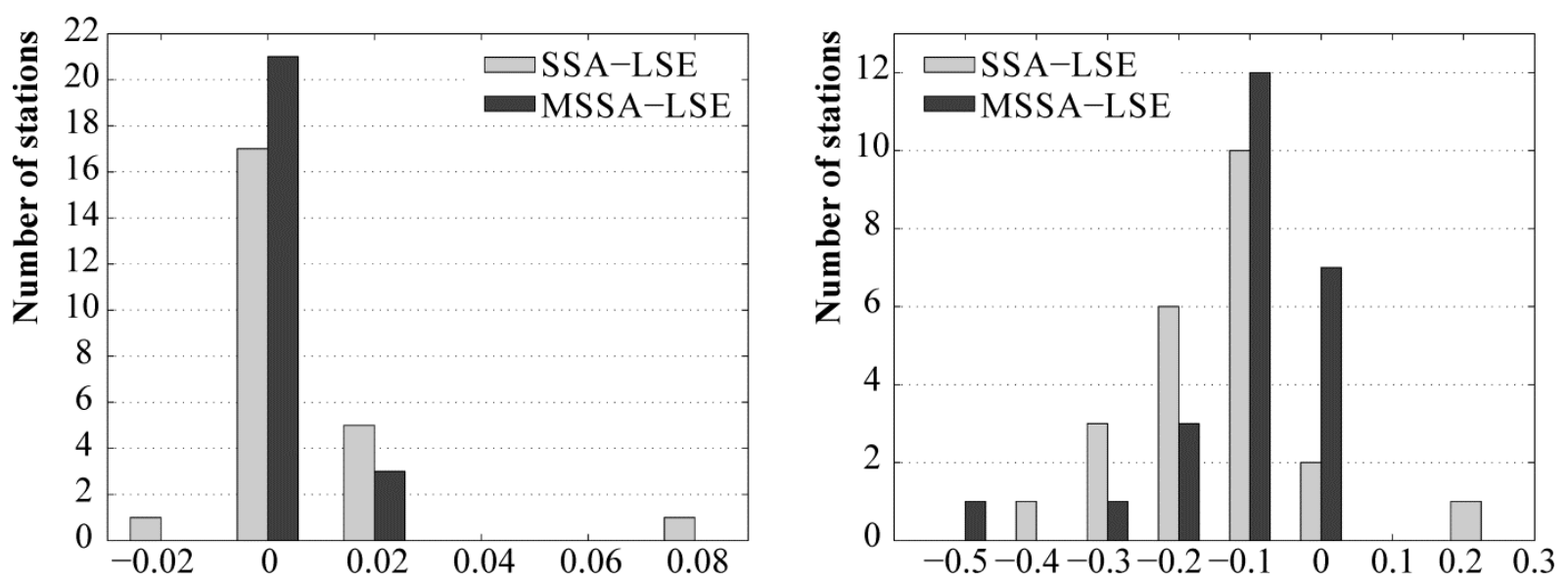

Fig. 10 Differences in spectral indices (left) and amplitude (right) of power-law character between SSA and MSSA in comparison to LSE. The closer the difference is to zero, the smaller is the difference between employed method and LSE.

\section{DISCUSSION AND CONCLUSIONS}

In this research, we delivered an approach to compare the Multichannel Singular Spectrum Analysis (MSSA) to Singular Spectrum Analysis (SSA) and to the widely used Least-Squares Estimation (LSE). Both LSE and SSA provide models estimated individually for each station, i.e. models estimated on station-by-station basis. Although LSE allows to model oscillations that are constant over time, SSA takes a step further and is an appropriate tool to estimate the oscillations that change over time. However, seasonal signals in GPS position time series may arise from common large-scale phenomena as e.g. hydrology. Due to above, in the following study, we discussed seasonal signals estimated with MSSA and compared them with methods used so far to determine the amplitudes of seasonal oscillations. This signal estimated with MSSA is a common seasonal oscillation that affects all GPS position time series employed to this analysis.

We proved that SSA affects the stochastic part of GPS data and artificially removes some part of power. Having estimated the percentage of variance explained by consecutive modes, we showed that some part of noise may be filtered out to neighbouring modes. The variance explained by annual signal estimated by SSA for individual station is approximately $3 \%$ lower than the one explained by MSSA curve. In the following research, we focused on estimates of common seasonal changes that affect a set of 24 IGS stations from Europe. When a joint seasonal signal is compared to the individual oscillations estimated separately for each of stations, we may be misled by the value of $3 \mathrm{~mm}$ when peakto-peak values are considered.

Time-varying curves estimated using MSSA were also compared to the ones estimated by SSA individually for each station. Although SSA curves match the time series better than MSSA curves, they contain lots of noise which is filtered out from neighbouring modes. When MSSA is applied, the only seasonal changes that are being modelled are the ones arising from large-scale phenomena. No station individual noise or local signal are being modelled.

As was already shown in previous papers, the stochastic part of GPS position time series is close to flicker noise (e.g. Klos et al., 2016). The main aim of this study is to model the common seasonal oscillations with no influence on the stochastic part of data. We should focus on modelling of real changes and remain the noise intact. We estimated the differences in spectral indices and amplitudes of power-law character estimated for residuals of LSE, SSA and MSSA using Hector software (Bos et al., 2013) (Fig. 10). The differences in spectral indices between MSSA and LSE are closer to zero than the ones between SSA and LSE. It means that MSSA curves are not affected by noise as much as the SSA curves are. SSA RCs contain some of the power which leaked through neighbouring modes. MSSA is not suffering from this problem. Estimates of common seasonal signal provide that we only model the timevarying large-scale phenomena and not local effects including noise and site-dependent variations. The differences in amplitudes of power-law behaviour between methods we employed and LSE were smaller after SSA was applied. It means that the standard deviation of data decreases when SSA-modelled curve was removed. So, we modelled and removed more variations with SSA method than with MSSA approach.

Finally, we estimated the uncertainties of velocities when seasonal oscillations were removed by using LSE, SSA and MSSA (Table 1). According to Bos et al. (2008) the velocity uncertainty has to be calculated using the values of spectral indices $(\kappa)$ and amplitudes $\left(A_{P L}\right)$ of coloured noise: 
Table 1 Vertical velocities and their uncertainties determined using different approaches.

\begin{tabular}{|c|c|c|c|c|c|c|}
\hline \multirow[b]{2}{*}{ Station } & \multicolumn{2}{|c|}{ LSE } & \multicolumn{2}{|c|}{ SSA } & \multicolumn{2}{|c|}{ MSSA } \\
\hline & $\begin{array}{l}\text { Velocity } \\
{[\mathrm{mm} / \mathrm{y}]}\end{array}$ & $\begin{array}{c}\text { Uncertainty } \\
{[\mathrm{mm} / \mathrm{y}]}\end{array}$ & $\begin{array}{l}\text { Velocity } \\
{[\mathrm{mm} / \mathrm{y}]}\end{array}$ & $\begin{array}{c}\text { Uncertainty } \\
{[\mathrm{mm} / \mathrm{y}]}\end{array}$ & $\begin{array}{l}\text { Velocity } \\
{[\mathrm{mm} / \mathrm{y}]}\end{array}$ & $\begin{array}{c}\text { Uncertainty } \\
{[\mathrm{mm} / \mathrm{y}]}\end{array}$ \\
\hline ACOR & -2.1 & 0.24 & -2.2 & 0.20 & -2.2 & 0.22 \\
\hline ALAC & -0.5 & 0.21 & -0.5 & 0.20 & -0.5 & 0.21 \\
\hline VIL0 & 2.3 & 0.70 & 2.4 & 0.69 & 2.5 & 0.69 \\
\hline CASC & -0.4 & 0.29 & -0.4 & 0.27 & -0.4 & 0.28 \\
\hline GLSV & 0.0 & 0.84 & 0.0 & 0.83 & 0.0 & 0.82 \\
\hline GOPE & 0.6 & 1.20 & 0.6 & 1.19 & 0.6 & 1.20 \\
\hline GRAZ & -0.2 & 0.69 & -0.3 & 0.67 & -0.2 & 0.68 \\
\hline HERS & -0.1 & 0.43 & -0.1 & 0.42 & -0.1 & 0.43 \\
\hline JOZE & 0.2 & 1.07 & 0.2 & 1.07 & 0.2 & 1.06 \\
\hline KIRU & 6.7 & 1.40 & 6.7 & 1.35 & 7.0 & 1.37 \\
\hline MARS & -1.0 & 0.22 & -1.0 & 0.22 & -1.0 & 0.22 \\
\hline MATE & 0.4 & 0.25 & 0.4 & 0.24 & 0.4 & 0.25 \\
\hline NOT1 & -1.1 & 0.21 & -1.1 & 0.20 & -1.1 & 0.20 \\
\hline ONSA & 2.9 & 1.11 & 2.9 & 1.09 & 3.0 & 1.10 \\
\hline POLV & 0.1 & 1.10 & 0.1 & 1.17 & 0.1 & 1.11 \\
\hline RIGA & 1.0 & 1.68 & 1.0 & 1.68 & 1.1 & 1.68 \\
\hline SASS & 0.2 & 0.95 & 0.2 & 0.94 & 0.2 & 0.95 \\
\hline SOFI & -0.1 & 0.20 & -0.1 & 0.19 & -0.1 & 0.19 \\
\hline TRO1 & 3.2 & 0.52 & 3.3 & 0.50 & 3.3 & 0.51 \\
\hline VIL0 & 8.5 & 2.41 & 8.7 & 2.27 & 8.9 & 2.30 \\
\hline VILL & -2.1 & 0.23 & -2.1 & 0.21 & -2.1 & 0.22 \\
\hline VIS0 & 2.8 & 2.46 & 2.9 & 2.44 & 3.0 & 2.45 \\
\hline WROC & -0.3 & 1.40 & -0.3 & 1.39 & -0.3 & 1.39 \\
\hline ZIMM & 0.4 & 0.54 & 0.4 & 0.52 & 0.4 & 0.53 \\
\hline
\end{tabular}

$$
\sigma_{v} \approx \pm \sqrt{\frac{A_{P L}^{2}}{\Delta T^{2-\frac{\kappa}{2}} \cdot \frac{\Gamma(3-\kappa) \cdot \Gamma(4-\kappa) \cdot(N-1)^{\kappa-3}}{\left[\Gamma\left(2-\frac{\kappa}{2}\right)\right]^{2}}}}
$$

with $\Delta T$ being time span.

The time-varying curves do not affect the values of velocities themselves. All changes in velocities between LSE, SSA and MSSA are within a formal error of velocity. The uncertainties of velocities when seasonal curves were estimated with LSE are always higher than the ones after SSA and MSSA, as LSE provides signals constant over time and does not include any time-changeability. The uncertainties estimated for residuals after SSA and MSSA are close to each other. However, uncertainties after SSA are always lower than the ones for residuals after MSSA. This is because SSA might remove some power which was filtered through the consecutive modes.

\section{ACKNOWLEDGMENTS}

We would like to thank the two anonymous reviewers for their suggestions and comments improving the manuscript.

GPS time series accessed from http://acc.igs.org/reprocess2.html on 2016-05-05.

Maps were drawn in the Generic Mapping Tool (GMT) (Wessel et al., 2013).
We modified Matlab-based algorithms written by Eric Breitenberger and downloaded from https://pantherfile.uwm.edu/kravtsov/www/downloads /KWCT2014/SSAMATLAB/mssa/.

This research was supported by the Military University of Technology, Faculty of Civil Engineering and Geodesy Young Scientists Development funds (798/2016).

\section{REFERENCES}

Akaike, H.: 1974, A new look at the statistical model identification. IEEE Trans. Autom. Control, 19 (6), 716-723. DOI: 10.1109/TAC.1974.1100705

Allen, M. and Robertson, A..: 1996, Distinguishing modulated oscillations from coloured noise in multivariate datasets. Clim. Dyn., 12 (11), 775-784. DOI: $10.1007 / \mathrm{s} 003820050142$

Altamimi, Z., Rebischung, P., Métivier, L. and Collilieux, X.: 2016, ITRF2014: A new release of the International Terrestrial Reference Frame modeling nonlinear station motions. J. Geophys. Res. Solid Earth, 121(8), 6109-6131.

DOI: $10.1002 / 2016 J B 013098$

Bennet, R.A.: 2008, Instantaneous deformation from continuous GPS: Contributions from quasi-periodic loads. Geophys. J. Int., 174(3), 1052-1064. DOI: $10.1111 /$ j.1365-246X.2008.03846.x

Blewitt, G., Lavalle, D., Clarke, P. and Nurutdinov, K.: 2001, A new global mode of Earth deformation: Seasonal cycle detected. Science, 294(5550), 23422345. DOI: $10.1126 /$ science. 1065328 
Bogusz, J. and Figurski, M.: 2014, Annual signals observed in regional GPS networks. Acta Geodyn. Geomater., 11, No. 2(174), 125-131. DOI: $10.13168 /$ AGG.2014.0003

Bogusz, J., Gruszczynska, M., Klos, A. and Gruszczynski, M.: 2016, Non-parametric estimation of seasonal variations in GPS-derived time series. Springer IAG Symposium, Series 146, Proceedings of the REFAG 2014. DOI: $10.1007 / 13452015191$

Bogusz, J. and Klos A.: 2016, On the significance of periodic signals in noise analysis of GPS station coordinates time series. GPS Solut., 20(4), 655-665. DOI: $10.1007 / \mathrm{s} 10291-015-0478-9$

Bos, M.S., Fernandes, R.M.S., Williams, S.D.P., and Bastos, L.: 2008, Fast error analysis of continuous GPS observations. J. Geod., 82, 157-166. DOI: $10.1007 / \mathrm{s} 00190-007-0165-\mathrm{x}$

Bos, M.S., Fernandes, R.M.S., Williams, S.D.P., and Bastos, L.: 2013, Fast error analysis of continuous GNSS observations with missing data. J. Geod., 87, 4, 351360. DOI: $10.1007 / \mathrm{s} 00190-012-0605-0$

Broomhead, D. and King, G.P.: 1986a, Extracting qualitative dynamics from experimental data. Physica D, 20, 2-3, 217-236.

DOI: $10.1016 / 0167-2789(86) 90031-X$

Broomhead, D. and King, G.P.: 1986b, On the qualitative analysis of experimental dynamical systems. Nonlinear Phenomena and Chaos, Adam Hilger, Bristol, 113-144.

Chen, Q., van Dam, T., Sneeuw, N., Collilieux, C., Weigelt, M. and Rebischung, P.: 2013, Singular spectrum analysis for modeling seasonal signal from GPS time series. J. Geodyn., 72, 25-35. DOI: 10.1016/j.jog.2013.05.005

Colebrook, J.M.: 1978, Continuous plankton records: Zooplankton and environment, Northeast Atlantic and North Sea. Oceanol. Acta, 1, 9-23.

Collilieux, X., Altamimi, Z., Coulot, D., Ray, J. and Sillard, P.: 2007, Comparison of very long baseline interferometry, GPS, and satellite laser ranging height residuals from ITRF2005 using spectral and correlation methods. J. Geophys. Res., 112, B12403. DOI: $10.1029 / 2007$ JB004933

Collilieux, X., van Dam, T., Ray, J., Coulot, D., Métivier, L. and Altamimi, Z.: 2012, Strategies to mitigate aliasing of loading signals while estimating GPS frame parameters. J. Geod., 86, 1-14. DOI: $10.1007 / \mathrm{s} 00190-011-0487-6$

Davis, J.L., Wernicke, B.P. and Tamisiea, M.E.: 2012, On seasonal signals in geodetic time series. J. Geophys. Res., 117(B1), B01403. DOI: 10.1029/2011JB008690

Dong, D., Fang, P., Bock, Y., Cheng, M.K. and Miyazaki, S.: 2002, Anatomy of apparent seasonal variations from GPS-derived site position time series. J. Geophys Res, 107, B4, 2075. DOI: 10.1029/2001JB000573

Dong, D., Fang, P., Bock, Y., Webb, F., Prawirodirdjo, L., Kedar, S. and Jamason, P.: 2006, Spatio-temporal filtering using principal component analysis and Karhunen-Loeve expansion approaches for regional GPS network analysis. J. Geophys. Res., 111, B03405. DOI: $10.1029 / 2005$ JB003806

Fraedrich, K.: 1986, Estimating dimensions of weather and climate attractors. J. Atmos. Sci,. 43, 419-432.

DOI: 10.1175/1520-0469(1986)043<0419:ETDOWA >2.0.CO.2

Freymueller, J.T.: 2009, Seasonal position variations and regional reference frame realization. In: Drewes, $\mathrm{H}$. (Eds.): Geodetic Reference Frames. Springer IAG
Symposium Series 134, 191-196.

DOI: $10.1007 / 978-3-642-00860-3 \quad 30$

Ghil, M., Allen, M.R., Dettinger, M.D., Ide K., Kondrashov, D., Mann, M.E., Robertson, A. W., Saunders, A., Tian, Y., Varadi, F. and Yiou, P.: 2002, Advanced spectral methods for climatic time series. Rev. Geophys., 40, 1, 1-41. DOI: 10.1029/2000RG000092

Golyandina, N. and Zhigljavsky, A.: 2013, Singular spectrum analysis for time series. Springer-Verlag Berlin Heidelberg. DOI: 10.1007/978-3-642-34913-3

Groth, A. and Ghil, M.: 2011, Multivariate singular spectrum analysis and the road to phase synchronization. Phys. Rev., 84, 3 . DOI: 10.1103/PhysRevE.84.036206

Gruszczynska, M., Klos, A., Gruszczynski, M. and Bogusz, J.: 2016, Investigation of time-changeable seasonal components in the GPS height time series: a case study for Central Europe. Acta Geodyn. Geomater., 13, No. 3(183), 281-289. DOI: $10.13168 /$ AGG.2016.0010

Hassani, H. and Mahmoudvand, R.: 2013, Multivariate singular spectrum analysis: A general view and new vector forecasting approach. International Journey of Energy and Statistics, 1, 1, 55-83. DOI: $10.1142 / \mathrm{S} 2335680413500051$

Hassani, H. and Zhigljavsky, A.: 2009, Singular spectrum analysis: methodology and application to economics data. J. Syst. Sci. Complex, 22, 3, 372-394.

DOI: $10.1007 / \mathrm{s} 11424-009-9171-9$

Jiang, W., Li, Z., van Dam, T. and Ding, W.: 2013, Comparative analysis of different environmental loading methods and their impacts on the GPS height time series. J. Geod., 87, 7, 687-703. DOI: $10.1007 / \mathrm{s} 00190-013-0642-3$

Karhunen, K.: 1946, Zur Spektraltheorie stochastischer Prozesse. Ann. Acad. Sci. Fenn. Ser. A1, Math. Phys., 34.

Khelifa, S., Kahlouche, S. and Belbachir, M. F.: 2012, Signal and noise separation in time series of DORIS station coordinates using wavelet and singular spectrum analysis. C. R. Geosci., 344, 334-348. DOI: $10.1016 /$ j.crte.2012.05.003

Kim, K.-Y., and Wu, Q.: 1999, A comparison study of EOF Techniques: Analysis of nonstationary data with periodic statistics. Journal of Climate, 12, 1, 185-199. DOI: 10.1175/1520-0442-12.1.185

Klos, A., Bogusz, J., Figurski, M. and Gruszczynski, M.: 2016, Error analysis for European IGS stations. Stud. Geophys. Geod., 60, 17-34.

DOI: $10.1007 / \mathrm{s} 11200-015-0828-7$

Klos, A., Gruszczynska, M., Bos, M.S., Boy, J.-P. and Bogusz, J.: 2017, Estimates of vertical velocity errors for IGS ITRF2014 stations by applying the improved singular spectrum analysis method and environmental loading models. Pure Appl. Geophys., 1-18. DOI: 10.1007/s00024-017-1494-1

Loève, M.: 1945, Fonctions alèatoires de second ordre. C. R. Acad. Sci. Paris, 220.

Penna, N.T. and Stewart, M.P.: 2003, Aliased tidal signatures in continuous GPS height time series. Geophys. Res. Lett., 30, 23, 2184. DOI: $10.1029 / 2003$ GL018828

Penna, N.T., King, M.A. and Stewart, M.P.: 2007, GPS height time series: Short-period origins of spurious long-period signals. J. Geophys. Res., 112, B2. DOI: 10.1029/2005JB004047 
Plaut, G. and Vautard, R.: 1994, Spells of low-frequency oscillations and weather regimes in the northern hemisphere. J. Atmos. Sci., 51, 2, 210-236. DOI: $10.1175 / 1520-0469(1994) 051<0210$ :SOLFOA $>2.0 . C O ; 2$

Rangelova, E., van der Wal, W., Braun, A., Sideris, M. G. and $\mathrm{Wu}, \mathrm{P}$. W.: 2007, Analysis of gravity recovery and climate experiment time-variable mass redistribution signals over North America by means of principal component analysis. J. Geophys. Res., 112, No. F03002. DOI: 10.1029/2006JF000615

Rangelova, E., Sideris, M.G. and Kim, J.W.: 2012, On the capabilities of the multi-channel singular spectrum method for extracting the main periodic and nonperiodic variability from weekly GRACE data. J. Geodyn., 54, 64-78. DOI: 10.1016/j.jog.2011.10.006

Rangelova, E., van der Wal, W., Sideris, M. G. and Wu, P.: 2010, Spatiotemporal analysis of the GRACE-derived mass variations in North America by means of multichannel singular spectrum analysis. In: Mertikas, S.P. (Eds.): Gravity, Geoid and Earth Observation, Springer IAG Symposium Series, 135, 539-546. DOI: $10.1007 / 978-3-642-10634-772$

Ray, J., Altamimi, Z., Collilieux, X. and van Dam, T.: 2008, Anomalous harmonics in the spectra of GPS position estimates. GPS Solut., 12, 1, 55-64.

DOI: 10.1007/s10291-007-0067-7

Raynaud, S., Yiou, P., Kleeman, R. and Speich, S.: 2005, Using MSSA to determine explicitly the oscillatory dynamics of weakly nonlinear climate systems. Nonlin. Processes Geophys., 12, 807-815.

DOI: $10.5194 /$ npg-12-807-2005

Rebischung, P., Altamimi, Z., Ray, J. and Garayt, B.: 2016, The IGS contribution to ITRF2014. J. Geod., 90, 7, 611-630. DOI: 10.1007/s00190-016-0897-6

Stewart, M., Penna, N. and Lichti, D. J.: 2005, Investigating the propagation mechanism of unmodelled systematic errors on coordinate time series estimated using least squares. J. Geod, 79, 8, 479-489. DOI: $10.1007 / \mathrm{s} 00190-005-0478-6$

Tesmer, V., Steigenberger, P., Rothacher, M., Boehm, J., and Meisel, B.: 2009, Annual deformation signals from homogeneously reprocessed VLBI and GPS height time series. J. Geod., 83, 10, 973-988. DOI: $10.1007 / \mathrm{s} 00190-009-0316-3$

Tregoning, P., Watson, C., Ramillien, G., McQueen, H. and Zhang, J.: 2009, Detecting hydrologic deformation using GRACE and GPS. Geophys. Res. Lett., 36, 15. DOI: 10.1029/2009GL038718

van Dam, T., Wahr, J. and Lavallée, D., 2007: A comparison of annual vertical crustal displacement from GPS and gravity recovery and climate experiment (GRACE) over Europe. J. Geophys. Res., 112, B03404. DOI: 10.1029/2006JB004335

van Dam, T., Wahr, J., Milly, P.C.D, Shmakin, A.B., Blewitt, G., Lavallée, D. and Larson, K.M.: 2001, Crustal displacements due to continental water loading. Geophys. Res. Lett., 28, 4, 651-654. DOI: $10.1029 / 2000$ GL012120

Vautard, R. and Ghil, M.: 1989, Singular spectrum analysis in nonlinear dynamics, with applications to paleoclimatic time series. Physica D, 35, 3, 395-424. DOI: 10.1016/0167-2789(89)90077-8

Vautard, R., Yiou, P. and Ghil, M.: 1992, Singular-spectrum analysis: a toolkit for short, noisy chaotic signals. Physica D, 58, 1-4, 95-125.

DOI: $10.1016 / 0167-2789(92) 90103-T$
Walwer, D., Calais, E. and Ghil, M.: 2016, Data-adaptive detection of transient deformation in geodetic networks. J. Geophys. Res. Solid Earth, 121, 3, 2129 2152. DOI: $10.1002 / 2015 J B 012424$

Welch, P.D.: 1967, The use of fast Fourier transform for the estimation of power spectra: A method based on time averaging over short, modified periodograms. IEEE Transactions on Audio Electroacoustics, 15, 2, 70-73. DOI: 10.1109/TAU.1967.1161901

Wessel, P., Smith, W.H.F., Scharroo, R., Luis, J. and Wobbe, F.: 2013, Generic mapping tools: Improved version released. EOS, Transactions, American Geophysical Union, 94, 45, 409-410. DOI: 10.1002/2013EO450001

Wu, X., Heflin, M.B., Ivins, E.R., Argus, D.F. and Webb, F.H.: 2003, Large-scale global surface mass variations inferred from GPS measurements of load-induced deformation. Geophys. Res. Lett., 30, 14. DOI: $10.2478 / \mathrm{s} 11600-011-0074-5$

$\mathrm{Xu}$, Ch. and Yue, D.: 2015, Monte Carlo SSA to detect time-variable seasonal oscillations from GPS-derived site position time series. Tectonophysics, 665, 118 126. DOI: $10.1016 /$ j.tecto.2015.09.029

$\mathrm{Xu}, \mathrm{Ch} .:$ 2016, Investigating mass loading contributors of seasonal oscillations in GPS observations using wavelet analysis. Pure Appl. Geophys., 173, 8, 27672775. DOI: $10.1007 / \mathrm{s} 00024-016-1301-4$

Zhang, J., Hassani, H., Xie, H. and Zhang, X.: 2014, Estimating multi-country prosperity index: a twodimensional singular spectrum analysis approach. J. Syst. Sci. Complex, 27, 1, 56-74. DOI: 10.1016/j.jog.2012.04.006

Zhu, C., Lu, Y., Shi, H. and Zhang, Z.: 2016, Spatial and temporal patterns of the inter-annual oscillations of glacier mass over Central Asia inferred from Gravity Recovery and Climate Experiment (GRACE) data. Journal of Arid Land, 9, 1, 87-97. DOI: $10.1007 / \mathrm{s} 40333-016-0021-\mathrm{z}$

Zerbini, S., Raicich, F., Errico, M. and Cappello, G.: 2013, An EOF and SVD analysis of interannual variability of GPS coordinates, environmental parameters and space gravity data. J. Geodyn., 67, 111-124. DOI: 10.1016/j.jog.2012.04.006 

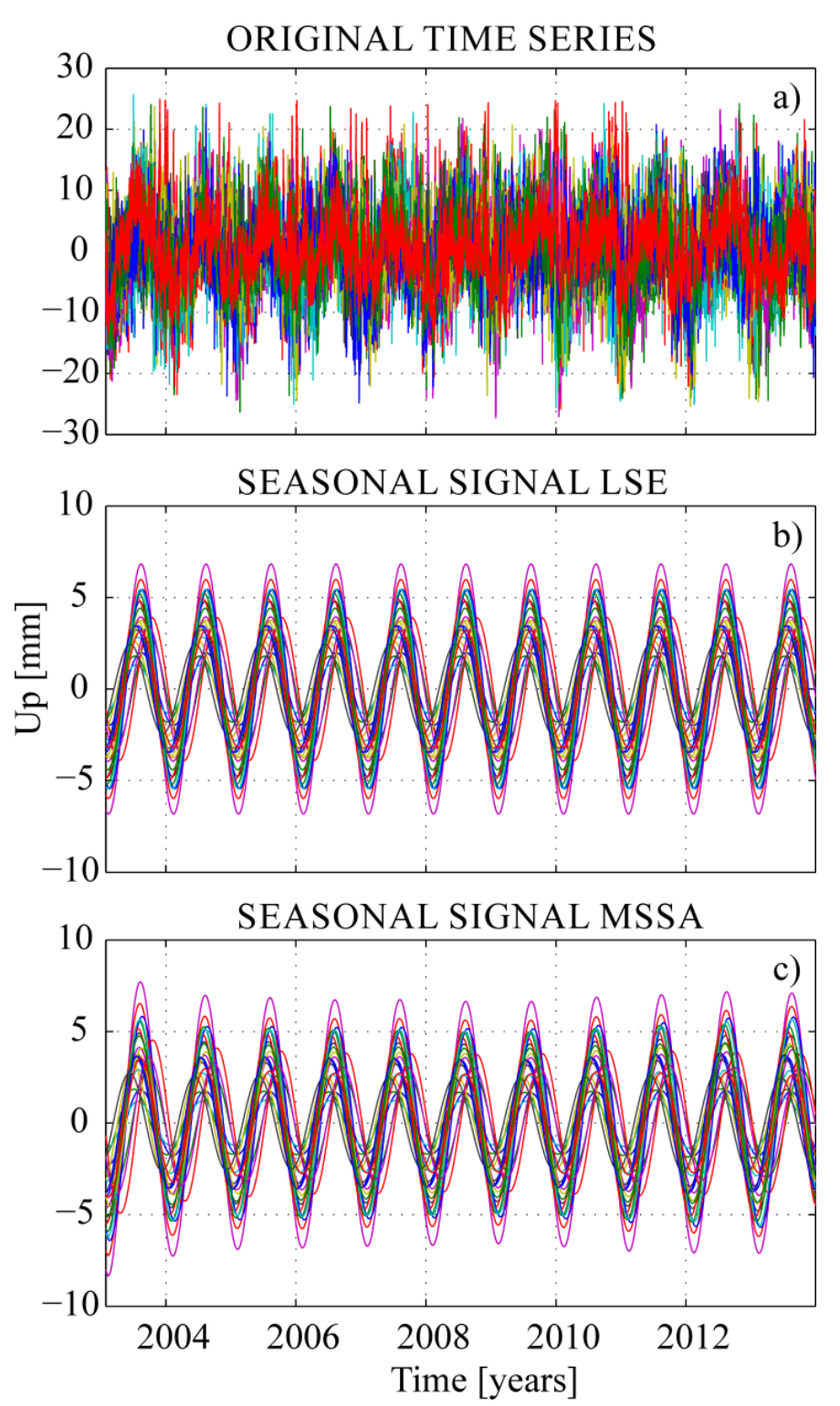
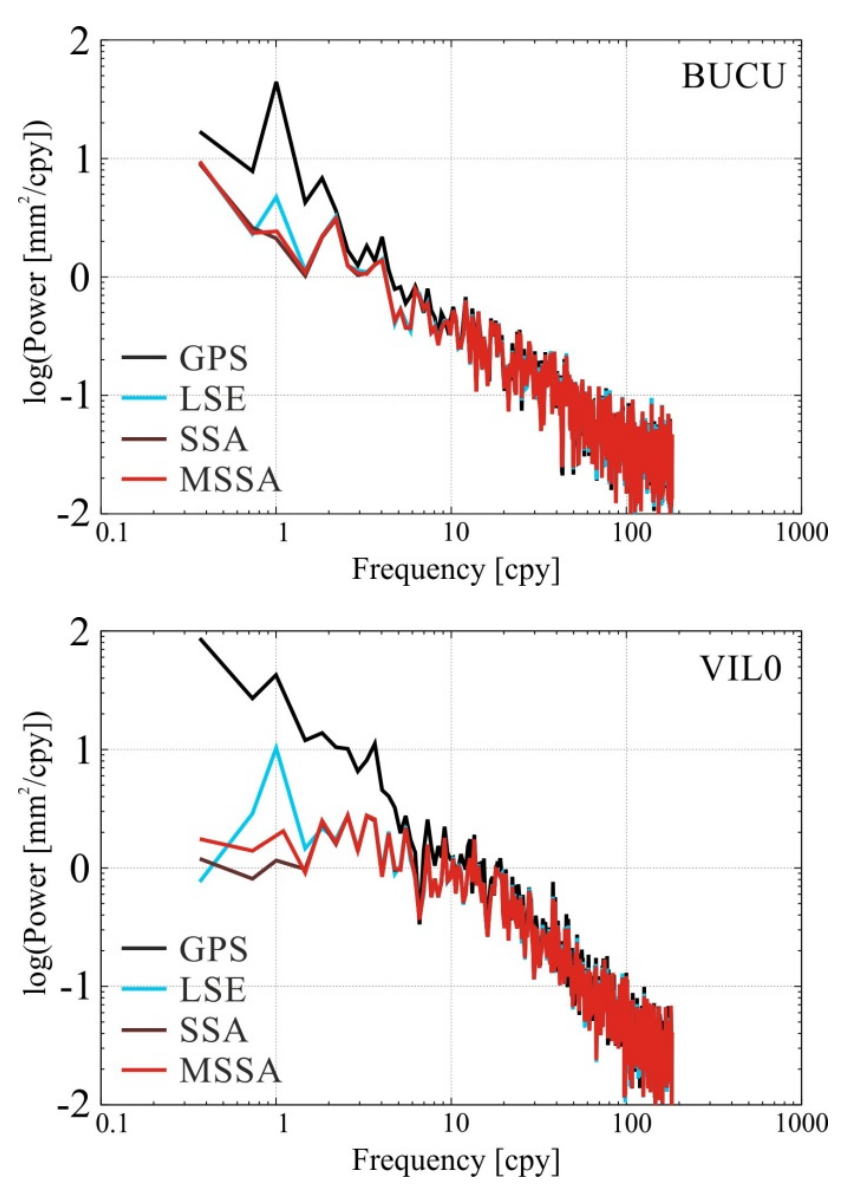

Fig. 9 Power Spectral Density estimated with Welch periodogram for BUCU and VIL0 station. Power of original data is plotted in black. Power of residuals after seasonal curves were removed with LSE, SSA and MSSA are plotted in blue, brown and red, respectively.

Fig. 6 Detrended GPS height time series (a) for all stations. Seasonal signals obtained using LSE (b) and MSSA (c) for individual stations are also presented. Same scales in (b) and (c) are used for a better comparison. 